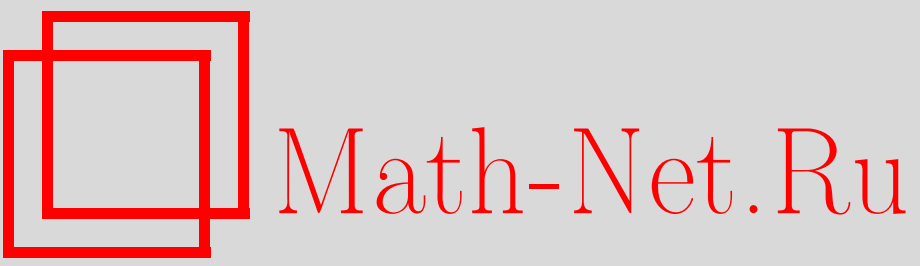

Ю. В. Муранов, Д. Реповш, Препятствия к перестройкам по паре многообразий, УМН, 1996, том 51, выпуск 4, 165-166

DOI: https://doi.org/10.4213/rm1006

Использование Общероссийского математического портала Math-Net.Ru подразумевает, что вы прочитали и согласны с пользовательским соглашением

http://www.mathnet.ru/rus/agreement

Параметры загрузки:

IP: 54.172 .240 .79

26 апреля 2023 г., 15:34:59 


\title{
ПРЕПЯТСТВИЯ К ПЕРЕСТРОЙКАМ ПО ПАРЕ МНОГООБРАЗИЙ
}

\author{
Ю. В. Муранов, Д. Реповш
}

Группы $L P_{*}$ препятствий к перестройкам по паре многообразий $X \subset Y$ были введены Уоллом геометрически (см. [1]), а затем Раницки было дано алгебраическое определение групп $L P_{*}$ аналогично группам препятствий к расщеплению $L S_{*}$ (см. [2]). Группы $L S_{n}$ и $L P_{n}$ функториально определяются универсальным отталкивающим квадратом $F$ фундаментальных групп с ориентацией и $n \bmod 4$. Пара $(Y, X)$ является парой Браудера-Ливси, если $X$ - одностороннее подмногообразие коразмерности 1 и отображения $\pi_{1}(\partial U) \rightarrow \pi_{1}(Y \backslash X), \pi_{1}(X) \rightarrow \pi_{1}(Y)$ - изоморфизмы, где $U$ - трубчатая окрестность $X$ в $Y$. Тогда имеют место изоморфизмы

$$
L S_{n}(F) \cong L N_{n}\left(\pi_{1}(Y \backslash X) \rightarrow \pi_{1}(Y)\right), \quad L P_{n}(F) \cong L_{n+1}\left(i^{!}\right)
$$

где $i^{!}: L_{n}\left(\pi_{1}(X) \rightarrow L_{n}\left(\pi_{1}(\partial U)\right)-\right.$ трансфер.

Если в этом случае условие изоморфизма ослабить до эпиморфизма, то квадрат $F$ называют геометрической диаграммой (см. [3]). Тогда по-прежнему имеется тесная связь между группами $L S_{*}(F)$ и $L$-группами групп, входящих в диаграмму (см. [4], [3]). Аналогичные связи в этом случае существуют и для групп $L P_{*}$.

Для геометрической диаграммы $F$ обозначим через $j_{-}^{!}$трансфер

$$
L_{n}\left(\pi_{1}(Y)^{-}\right) \rightarrow L_{n}\left(\pi_{1}(Y \backslash X)\right),
$$

где знак "-" обозначает, что группа рассматривается с измененной вне образа $j$ ориентацией. Обозначим $\delta_{n}$ композицию

$$
L_{n+1}\left(\pi_{1}(\partial U) \rightarrow \pi_{1}(Y \backslash X)\right) \rightarrow L_{n}\left(\pi_{1}(\partial U)\right) \rightarrow L_{n}\left(i^{!}\right)
$$

отображения из относительной точной последовательности для вложения $\pi_{1}(\partial U) \rightarrow \pi_{1}(Y \backslash X)$ и отображения из относительной точной последовательности для отображения $i^{!}$. Аналогично, определим отображение $\delta_{n}^{\prime}$ как композицию

$$
L_{n+1}\left(j_{-}^{!}\right) \rightarrow L_{n}\left(\pi_{1}(Y)\right) \rightarrow L_{n}\left(\pi_{1}(X) \rightarrow \pi_{1}(Y)\right) .
$$

Теорема 1. Если F- геометрическал диаграмма групп, то имеют место следующие длинные точные последовательности

$$
\begin{aligned}
& \left.\longrightarrow L_{n+1}\left(\pi_{1}(\partial U)\right) \longrightarrow \pi_{1}(Y \backslash X)\right) \stackrel{\delta_{n}}{\longrightarrow} L_{n}\left(i^{!}\right) \longrightarrow L P_{n-1}(F) \longrightarrow \\
& \longrightarrow L_{n+1}\left(j_{-}^{!}\right) \stackrel{\delta_{n}^{\prime}}{\longrightarrow} L_{n}\left(\pi_{1}(X) \rightarrow \pi_{1}(Y)\right) \longrightarrow L P_{n-1}(F) \longrightarrow
\end{aligned}
$$

Доказательство следует из коммутативной диаграммы спектров, которая продолжается корасслоениями:

$$
F^{!}=\begin{array}{ccc}
\boldsymbol{L}\left(\pi_{1}(\partial U)\right) & \longrightarrow \boldsymbol{L}\left(\pi_{1}(Y \backslash X)\right) \\
\uparrow_{i} ! & & \uparrow j_{-}^{!} \\
\boldsymbol{L}\left(\pi_{1}(X)\right) & \longrightarrow & \boldsymbol{L}\left(\pi_{1}(Y)\right)
\end{array}
$$

оставаясь гомотопически коммутативной. Для $\boldsymbol{L}$-спектров мы полагаем, что $\pi_{n}(\boldsymbol{L}(*))=L_{n}(*)$ и $\Omega \boldsymbol{L}_{n+1}=\boldsymbol{L}_{n}$, где $\boldsymbol{L}_{n}$ - симплициальные множества, задающие спектр.

Диаграмма $F^{!}$позволяет, аналогично [4], определить спектры $\boldsymbol{L}\left(F^{!}\right)$и $\boldsymbol{L} P(F)$. При этом $\pi_{n}(\boldsymbol{L} P(F))=L P_{n}(F)$.

Работа первого автора выполнена при финансовой поддержке Российского фонда фундаментальных исследований (грант № 93-011-1402) и Международного научного фонда (NFU000).

Работа второго автора выполнена при финансовой поддержке министерства науки и техники Республики Словения (грант № Р1-0214-101-95). 
ТеОРема 2. Имеют место универсальные квадраты спектров:

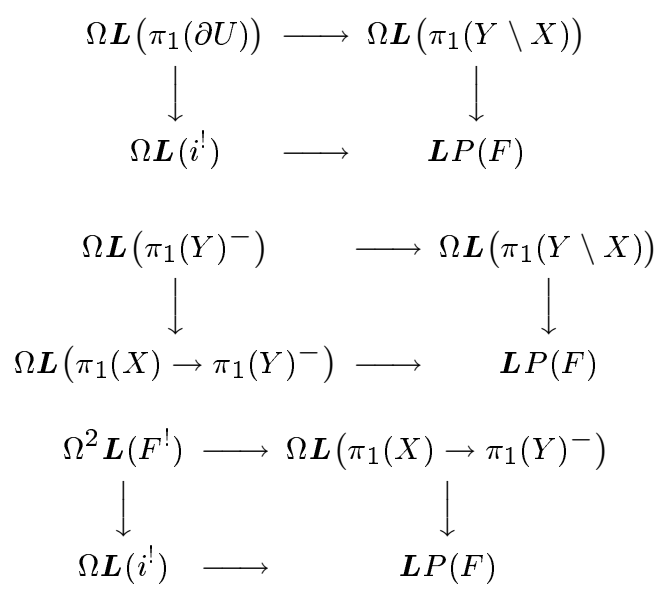

Гомотопические длинные точные последовательности отображений универсальных квадратов дают три косы Левина, связивающие группы $L P *(F)$ с L-группами.

\section{СПИСОК ЛИТЕРАТУРЫ}

[1] Wall C. T. C. Surgery on Compact Manifolds. London: Academic Press, 1970. [2] Ranicki A. Exact sequences in the algebraic theory of surgery. Princeton: Princeton Univ. Press 1981 (Matem. Notes, V. 26). [3] Ахметьев П. М. Задача расщепления гомотопических эквивалентностей // Дис. ... канд. физ.-матем. наук. М.: МИАН, 1988. [4] Муранов Ю. В. // УМН. 1995. Т. 50. № 1. С. 205-206.

Математический институт им. В. А. Стеклова РАН,

Принято редколлегией Москва, Россия;

18.04.1996

Люблянский университет,

Институт математики, физики и механики,

Любляна, Словения 